\title{
Editorial
}

\section{Plagiarism and detection}

\author{
C. Barry Carter $^{1, *}$ and Christopher F. Blanford ${ }^{2}$
}

${ }^{1}$ Department of Materials Science \& Engineering and Department of Chemical \& Biomolecular Engineering, University of Connecticut, Storrs, CT 06239, USA

${ }^{2}$ School of Materials and Manchester Institute of Biotechnology, University of Manchester, 131 Princess Street, Manchester M1 7DN, UK

Received: 17 April 2016

Published online:

28 April 2016

(C) Springer Science+Business

Media New York 2016

The topic of plagiarism is never far away from an Editor's mind. Plagiarism undermines the whole of scientific publishing: it increases the reviewing burden on overloaded researchers, dilutes the quality of information available in an increasing sea of scientific literature, and mars journals' reputations as reliable curators of scientific knowledge.

In the past, it was almost impossible to catch plagiarism before publication. Occasionally readers would inform an Editor of such a case, having read both papers because the two papers were on the same subject. Sometimes papers were published twice by accident, even by distinguished authors. Today some authors will submit the same manuscript to more than one journal and try to withdraw one if the other is accepted first. That is not plagiarism, just dishonesty. A good example of such double publication is given by the publications of
Sarkar et al. in J Mater Sci (2007) 42:1847-1855 and Ceramics International (2007) 33: 1275-1282. The title and some of the words have been changed but the figures are almost identical in both the papers. Some of the same figures appear in the earlier (by submission date) 2007 paper by Sarkar, Adak and Mitra, which went through the review process at the same time. The point to make is that the actions of these authors are now easily available for all to see.

This Journal has seen a recent sharp rise in partial plagiarism, in which significant blocks of text from others are reused verbatim, and the related 'text recycling' (also known as self-plagiarism), where authors reuse passages of their own text. We Editors are guided by the codes of conduct and best practice developed by the Committee on Publication Ethics (COPE), of which our publisher, Springer Nature, is a

Address correspondence to E-mail: : cbarrycarter@gmail.com 
member. ${ }^{1}$ We do not automatically presume malicious intent when there is evidence of text reuse. Overlapping blocks of text could either be the result of outright dishonesty (some cases certainly are) or could be the result of authors using 'model' papers to overcome language difficulties for non-native-English speakers or recycling text from the authors' previous manuscripts. We understand that descriptions on methods sections are likely to use similar phrases, but when the copied sections appear in the results, we are especially concerned. One comment received from an author when confronted with the fact that he had copied a section almost verbatim: "we always appreciated Professor X's work and couldn't explain the procedure better than he did in his paper". If authors quote text that has been published elsewhere, then they should use quotation marks and cite the source. The referees can decide if there is enough new text to justify publication.

Journals and publishers have different policies for dealing with plagiarized work after publication. Some may remove the plagiarizing paper so that there is no evidence that the journal was tricked into publishing plagiarized work. Unfortunately this also removes the evidence of the misdeed. The Journal of Materials Science, like many other journals, flags the offending paper with a plagiarized watermark. The DOI remains the same-the plagiarized paper is a permanent record of the misdeed.

So how do we spot plagiarism before publication at the Journal of Materials Science?

Every new and revised manuscript sent to the Journal is checked automatically by CrossCheck by iThenticate and assigned a similarity score, given as a percentage, of how much of the text in the submission overlaps with previously published text from tens of thousands of journals, billions of web pages, and millions of other content items. Many academic users will be familiar with its sibling, TurnItIn. Segments in a submission that match passages from indexed sources are color-coded and related to the published work. Incidentally, for only a nominal cost, authors can quickly submit their manuscripts to iThenticate to see if there are problems.

\footnotetext{
${ }^{1}$ Details are given in Springer Nature's policy document on this subject (https://www.springer.com/gp/authors-editors/ editors / publishing-ethics-for-journals /4176).
}

At least one Editor always looks over articles with high similarity scores. Typically, the Editor-in-Chief personally assesses every manuscript that scores more than $\sim 30 \%$. The individual Editors then assess every paper that they receive. For example, a paper scoring $25 \%$ on the 'similarity' test might have literally copied $20 \%$ from a single previous paper. Other times, the References section may not have been not automatically excluded, because for example, it was not labeled 'References', which would lead to inflated scores. When considering whether to sending out a manuscript for review, the Editors conduct a literature search on the submission's topic, which sometimes reveals cases of 'article spinning'-automated rewriting of published text to generate a 'new' submission designed to foil plagiarism checkers.

Some of the authors of the 'new' paper might be completely unaware that their co-author had actually copied or spun text from an earlier paper, which is a real cause for concern. Submissions by 'authors' who have previously extensively copied papers receive additional scrutiny.

At present, the editors of all reputable journals are monitoring this phenomenon and over 500 of them are using iThenticate. The solution to this problem is not obvious: the rise in the use of plagiarism-detection software has led to an arms race, with new manual and automated methods to defeat the software. (Search for 'how to beat TurnItIn' for dozens of examples.) Unfortunately, we have already seen the next stage, in which the manuscript is carefully reworded to circumvent detection but the diagrams remain the same as in the original paper. In the future, deposition of datasets related to a publication will be a universal requirement; these will certainly be automatically scrutinized for duplication and falsification. In the meantime, the Journal continues to rely on the collective experience of its $20+$ researchactive Editors to spot suspicious papers and root out plagiarism so the Journal of Materials Science can maintain its 50-year-old reputation as a steward of high-quality materials science research. 